\title{
High-Sensitivity MEMS Pressure Sensor Utilizing Bipolar Junction Transistor with Temperature Compensation
}

\author{
M. Basov * \\ Dukhov Automatics Research Institute (VNIIA), Moscow 127055, Russian Federation \\ *Corresponding author. E-mail address: engineerbasovm@gmail.com (M. Basov)
}

\begin{abstract}
The paper presents MEMS pressure sensor chip utilizing novel electrical circuit with bipolar-junction transistor-based (BJT) differential amplifier with negative feedback loop (PDA-NFL). Pressure sensor chips with two circuits have been manufactured and tested: the first chip uses circuit with vertical n-p-n (V-NPN) BJTs and the second - circuit with horizontal p-n-p (L-PNP) BJTs. The demonstrated approach allows for increase of pressure sensitivity while keeping the same chip size. It also can be used for chip size reduction and increase of pressure overload capability while maintaining the same pressure sensitivity. Significant reduction of both noise and temperature instability of output signal has been demonstrated using transistor amplifier with negative feedback loop.
\end{abstract}

This is a green open access article under the CC BY license.

Keywords: MEMS, pressure sensor, differential amplifier, bipolar junction transistor

\section{Introduction}

MEMS pressure sensors are widely used in modern electronic devices. Large percentage of measurements in industrial applications (automotive, process control, aviation, space, medical, power plants) and in R\&D (fluid mechanics, robots, biophysics, acoustics, geophysics and others), are linked to measurements of pressure, flow, level, consumption of fluid and made with help of pressure sensors. As it can be seen from Fig. 1, pressure sensor market (after first-level packaging) is currently growing with CAGR of 3.8\% and will reach $\$ 2.0 \mathrm{~B}$ in 2023 (vs $\$ 1.6 \mathrm{~B}$ in 2016) [1]. Majority of pressure sensors utilize either piezoresistive or capacitive principle of operation. Growing demand for pressure sensors stimulates development of novel types of MEMS pressure sensor chips with improved performance, including higher sensitivity, better accuracy, and smaller die size.

A promising direction in development of high-performance piezoresistive pressure sensors is on-chip integration of piezoresistors with some transistor-based circuits for pressure sensitivity increase. Both MOS and bipolar junction transistors (BJT) transistors can be used either as pressure sensitive elements or can be included as non-pressure sensitive elements in electrical circuits of piezoresistive sensors. This paper discusses pressure sensor chips utilizing circuits with BJTs.

It is worth mentioning that devices using MOS transistors in piezoresistive sensors are often based either on combining pressure-sensitive transistors in Wheatstone bridge 


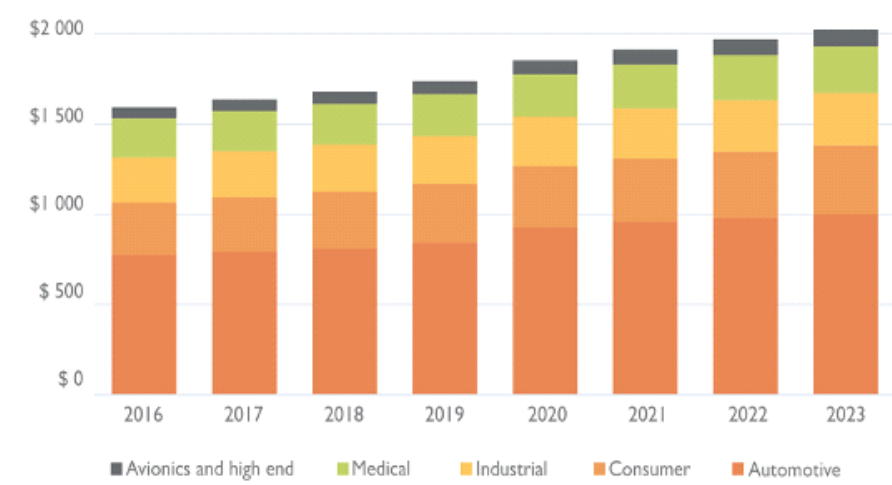

Fig. 1. MEMS pressure sensors sales growth in 2016-2023 [1].

circuit $[2,3]$ or on a single MOS transistor [4,5] having channel resistance modulated by applied pressure. Main advantage of such approach is related to smaller size of MOS transistor compared to size of piezoresistors. MOS transistors can be located in small areas where applied pressure causes the maximum stress. Despite the fact that significant increase of pressure sensitivity compared to piezoresistive sensors has not been reported, MOS transistors can be useful in design of small size piezoresistive sensing elements.

First studies of piezoeffect in BJT performed in 1960-70s by W. Rindner, Y. Kanda R. Edwards, and A.L. Polyakova [6-9] were not related to development of pressure sensors. Piezoeffect was evaluated based on modulation of I-V curves of Si and Ge transistors by pressure applied with help of a needle. In these tests, mechanical stress could be as high as $1 \mathrm{GPa}$. Later, use of BJT in pressure sensor chips was proposed by V.I. Vaganov. The work was done in Moscow Physics-Engineering institute (MPhEI), currently National Research Nuclear University MPhEI. Vaganov and his colleagues studied differential amplifiers combining BJT and piezoresistors in pressure sensors. Both transistors and piezoresistors connected to their bases and collectors were located on diaphragms and were sensitive to pressure $[10,11]$. The same circuit has been selected in this work as a prototype for further study. The results reported by Vaganov et al. are in good agreement with publication by Fruett F. и Meijer G.C.M. (Delft University of Technology), who described stress-sensitive behavior of elements with p-n junctions and called it "piezojunction effect" [12].

It seems that transistor circuits in piezoresistive sensors did not attract attention because of several factors. Some of the factors are listed below.

- Piezoresistive sensors generate high output signal of tens or hundreds of milliVolts, which can be used without on-chip signal conditioning.

- On-chip integration of a simple transistor amplifier with piezoresistors that can be accomplished in MEMS fabs was not considered useful as it does not eliminate the need for sensor signal conditioning by external ASIC or microcontroller. At the same time, on-chip integration of pressure sensor with CMOS electronics in semiconductor fabs requires new processes (silicon micromachining, double-side alignment) and use double-side polished substrates. This leads to a need for new equipment and modification of existing equipment, which may not be practical at the current market size for piezoresistive sensors. 
- Adding on-chip amplification circuits to piezoresistive Wheatstone bridge results in a different behavior of the sensor output in operating temperature range and increases output signal noise compared to just Wheatstone bridge - both factors are undesirable.

- Addressing the above challenge requires collaboration of MEMS and analog circuit design engineers, which is a rear combination in the field of piezoresistive sensors because Wheatstone bridge circuit is very simple and design of new sensor chips is often done without use of IP related to analog circuits.

However, on-chip integration of transistor amplifiers can bring some important advantages to piezoresistive pressure sensors. It allows for increase of pressure sensitivity, which, in turn, leads to improvement of pressure sensor performance and/or reducing die size while maintaining the same level of output signal. Sensitivity increase can be "exchanged" on higher pressure overload capability or on tighter distribution of parameters or smaller temperature dependence of output signal. Another advantage is ability to offer sensors with very low operating pressure range (0.5-5.0 $\mathrm{kPa}$ ) or very small size. In both cases, the limitation is related to very low output signal. So far, most of work was focused on new microstructure design [13-16]. On-chip amplification can be important to generate signal that can be transferred for signal conditioning without significant errors.

Therefore, use of BJTs either as stress-sensitive components or as elements of stress-sensitive circuits is a promising direction in development of MEMS pressure sensors.

This paper discusses two circuits that can be used in piezoresistive sensors: 1) circuit with differential amplifier on BJTs (PDA circuit) and 2) circuit with differential amplifier on BJTs having negative feedback loop (PDA-NFL circuit).

\section{Pressure Sensor Chip with PDA Circuit}

Pressure sensing element with PDA circuit (later referred as die or pressure sensor die) has size of 4.0 x 4.0 x 0.4 Mm. It has been manufactured using (100) p-type Si wafers with n-type epitaxial layer. The pressure sensor die has square diaphragm $(2.0 \mathrm{~mm} \times$ $2.0 \mathrm{~mm} \times 0.028 \mathrm{~mm})$ with square rigid island $(1.2 \mathrm{~mm} \times 1.2 \mathrm{~mm})$ in the center. The rigid island works as a stress concentrator. Mechanical structure is designed for pressure range of $60 \mathrm{kPa}$ and pressure non-linearity spec of $0.15 \%$. Electrical circuit formed on the pressure sensor die is shown in Fig. 2. The circuit utilizes differential amplifier based on two n-p-n BJTs having vertical structure (V-NPN). The transistors are located on the diaphragm in the areas with the maximum mechanical stress [17, 18]. Four piezoresistors connected to bases and collectors of both transistors also change their resistance in response to pressure as shown by arrows in Fig. 2.

Resistors are formed by combination of low-doped $\mathrm{P}^{-}$areas (sheet resistance $200 \mathrm{Ohm} / \mathrm{sq}$.) and high-doped $\mathrm{P}^{+}$areas (sheet resistance $20 \mathrm{Ohm} / \mathrm{sq}$.). Resistors $\mathrm{R}_{\mathrm{c} 1}$ and $\mathrm{R}_{\mathrm{c} 2}$ are $0.5 \mathrm{kOhm}$ and resistors $\mathrm{R}_{\mathrm{b} 1}$ and $\mathrm{R}_{\mathrm{b} 2}$ are $5.4 \mathrm{kOhm}$. Vertical NPN transistors $\mathrm{T}_{1}$ and $\mathrm{T}_{2}$ are formed by low-doped $\mathrm{P}^{-}$areas and high-doped $\mathrm{N}^{+}$areas. When the circuit is powered by voltage $U_{\text {sup }}=2.0 \mathrm{~V}$, then transistors $T_{1}$ and $T_{2}$ have gain coefficient $\beta$ of about 100 at 


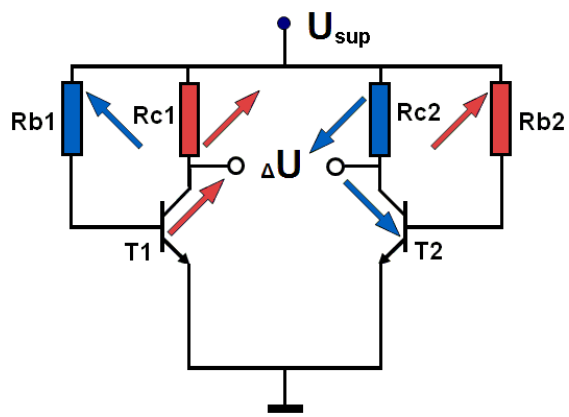

a)

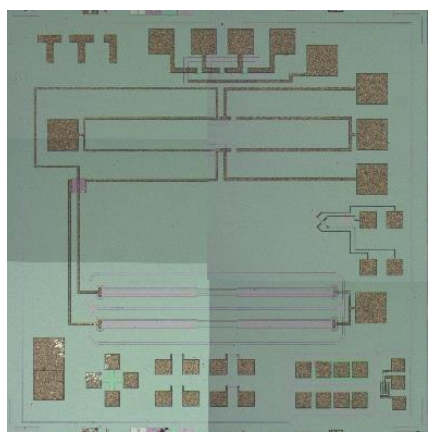

b)

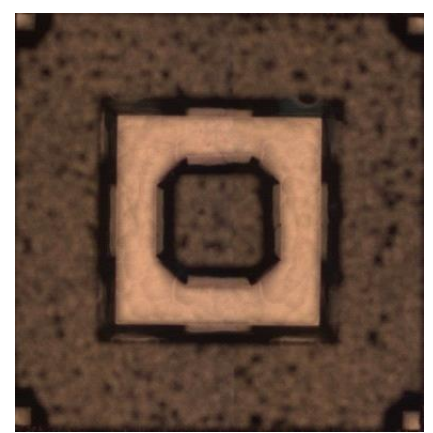

c)

Fig. 2. Pressure sensor chip with PDA circuit: a) electrical circuit, b) top view, c) back view.

base-emitter current of $14 \mu \mathrm{A}$. Isolation diffusion areas $\left(\mathrm{P}^{+}\right)$extending through full thickness of epi-layer are used to electrically isolate transistors and piezoresistors. P-substrate is connected to ground. Difference in electrical potentials of collectors of transistors $T_{1}$ and $T_{2}$ is used as output signal $\Delta U$ of the sensor.

Sensitivity of the sensor chip with PDA circuit was compared to sensitivity of pressure sensor chip (TM sensor) fabricated using the same process and having the same mechanical structure but utilizing Wheatstone bridge circuit formed by just four piezoresistors. During testing, pressure was applied from the cavity side (back side). Fig. 3(a) shows dependence of average pressure sensitivity on supply voltage for sensors with PDA circuit. It can be seen that sensitivity rapidly increases with increase of supply voltage from $0.5 \mathrm{~V}$ to $2.0 \mathrm{~V}$ and practically does not change with further increase of supply voltage. Fig. $3 b$ shows transduction characteristics of TM sensor and sensor with PDA circuit. As it can be seen, sensor with PDA circuit has pressure sensitivity of $0.66 \mathrm{mV} / \mathrm{V} / \mathrm{kPa}$. It is 2.2 times higher than sensitivity of TM sensor.

Testing showed that pressure sensor chip with PDA circuit has some drawbacks, including high level of noise $\Delta \mathrm{U}_{\text {noise }}=300 \mu \mathrm{V}$, large temperature errors and instability. Testing was performed in $-30 \ldots+80{ }^{\circ} \mathrm{C}$ temperature range and average parameters were calculated for the pressure range of $\mathrm{P}=60 \mathrm{kPa}$.

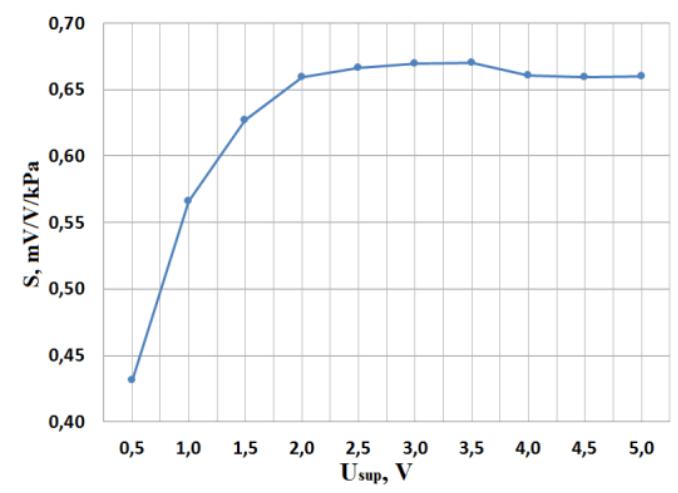

a)

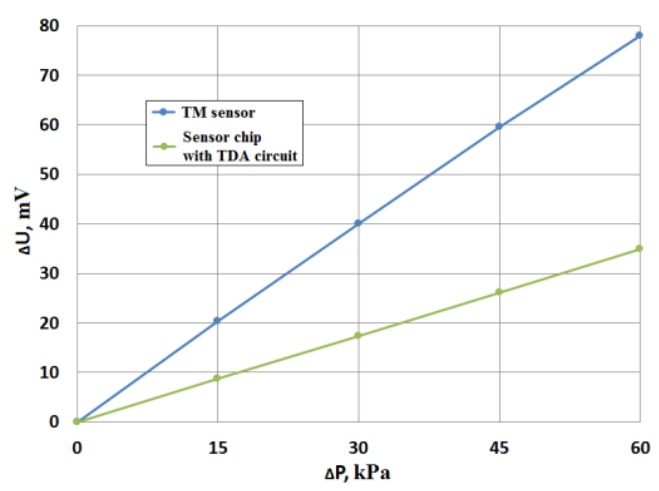

b)

Fig. 3. Dependence of pressure sensitivity on voltage supply for sensor with PDA circuit (a), dependence of sensor output on pressure for TM sensor and sensor with PDA circuit (b). 
- Thermal hysteresis of zero offset is $1.03 \% \mathrm{FS}$,

- Thermal hysteresis of span $0.33 \% \mathrm{FS}$,

- Temperature coefficient of zero offset $0.25 \% \mathrm{FS} /{ }^{\circ} \mathrm{C}$, and

- Temperature coefficient of sensitivity $=0.32 \% \mathrm{FS} /{ }^{\circ} \mathrm{C}$.

It is believed that large sensor errors are linked to properties of bipolar junction transistors and to the circuit used in the sensor.

- BJTs have higher noise compared to piezoresistors due to flicker noise or 1/f noise caused by carrier generation / recombination in base areas of transistors.

- Base potentials have large temperature dependence. Small temperature change or small temperature difference between two transistors may result in significant change of the output signal.

\section{Pressure Sensor Chip with PDA-NFL Circuit}

PDA-NFL circuit shown in Fig. 4a is a modification of PDA circuit. PDA-NFL circuit addresses the above-mentioned drawbacks of PDA circuit. Two types of BJTs have been used: vertical n-p-n transistors (V-NPN) and horizontal (lateral) p-n-p transistors (L-PNP). Sensor chip with circuit utilizing V-NPN transistors is shown in Fig. $4 \mathrm{~b}$ and sensor chip with circuit utilizing L-PNP transistors is shown in Fig. 4c. The circuit used in sensor with L-PNP transistors is similar to the one shown in Fig. 4a. The difference is in

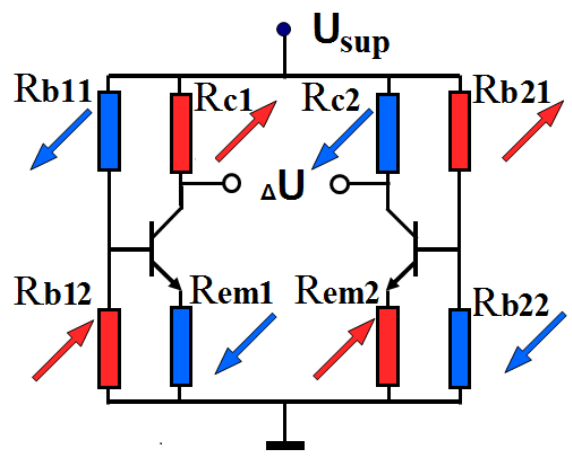

a)

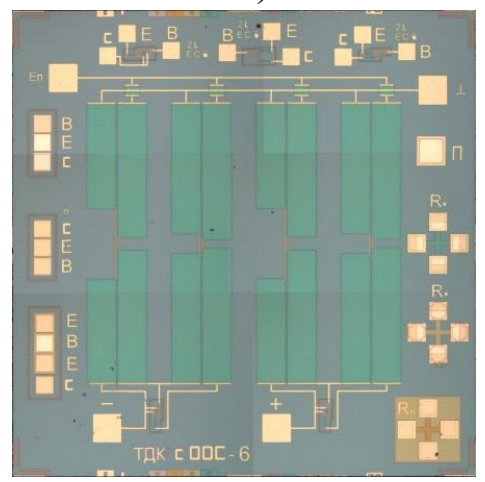

c)

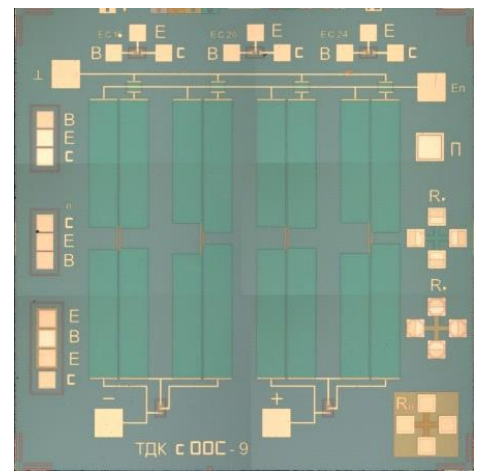

b)

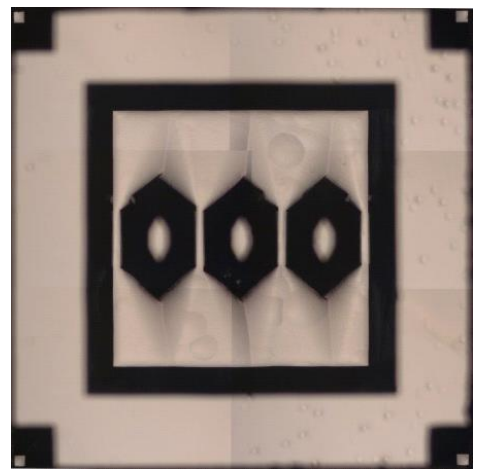

d)

Fig. 4. Sensor chip with PDA-NFL circuit: a) PDA-NFL circuit with V-NPN BJTs, b) top view of chip with V-NPN BJT circuit, c) top view of chip with L-PNP BJT circuit, d) back side view. 
polarity of power supply $U_{\text {sup }}$ (see Fig. 5). Noise reduction can be achieved by decreasing width of the base in V-NPN transistors and by using two elongated collector areas surrounding emitter and increasing area of $\mathrm{p}$-n junction conducting current in case of L-PNP transistors [19]. Negative feedback loop and optimized values of the resistors allows for significant decrease of unwanted dependence of base potential on temperature. In addition, the sensors are expected to have higher pressure sensitivity due to combining a larger number of piezoresistors in one circuit with BJTs.

At the design phase, electrical circuits were studied using both analytical methods and modeling in NI Multisim software. An important result is that placing BJTs on the diaphragm provides only $1 \%-5 \%$ increase of pressure sensitivity. The results are illustrated in Fig. 5.

The developed mathematical model uses known dependences of gain in BJT on stress [17] and parameters of piezo-junction effect [12]. From Fig. 5 it can be seen that placing BJTs on thin portion of the diaphragm does not offer significant advantages in pressure sensitivity. However, placing BJTs on the diaphragm makes die layout much more complicated because all elements should be combined in a relatively small area between the rigid islands, where the maximum stress is generated by applied pressure. Besides that, all circuit elements should be connected without using aluminum metal lines

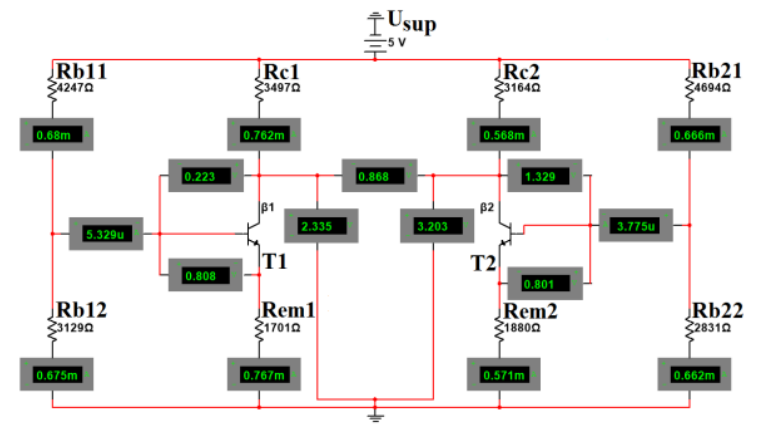

a)

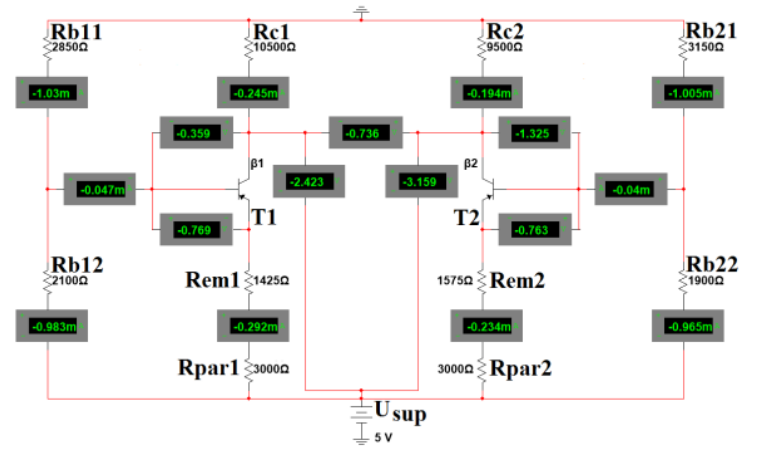

c)

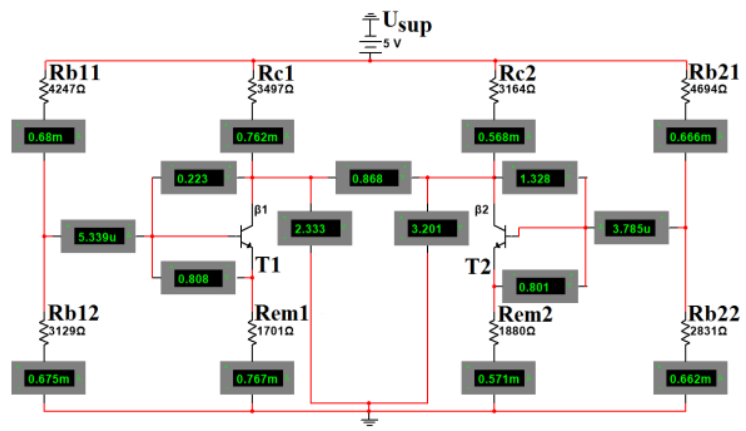

b)

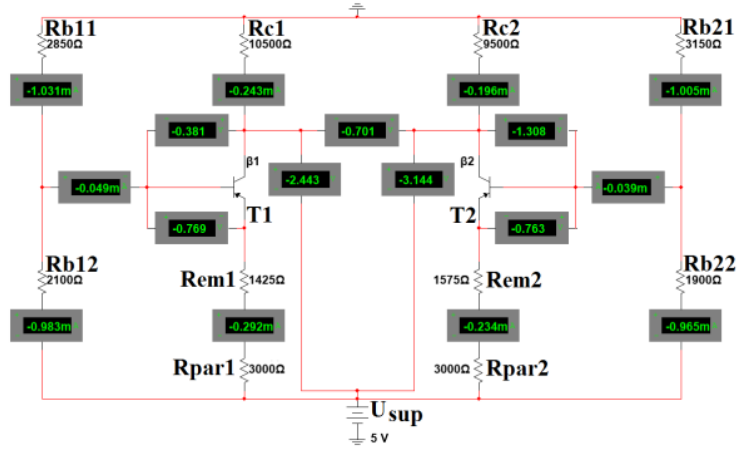

d)

Fig. 5. Resistors and currents in PDA-NFL circuit from modeling in NI Multisim (pressure applied): a) circuit with V-NPN BJTs located on the diaphragm; b) circuit with V-NPN BJTs located on the frame; c) circuit with L-PNP BJTs located on the diaphragm; d) circuit with L-PNP BJTs located on the frame. 
to avoid thermal hysteresis caused by big difference in coefficients of thermal expansion (CTE) of aluminum and silicon. Therefore, transistors in PDA-NFL circuit were placed on the thick frame in both versions of the sensor chip - with V-NPN and L-PNP circuits.

Sensor chip with PDA-NFL circuit has similar size and fabricated on the same type of wafers with epitaxial layer as the sensor chip with PDA circuit. The chip has square $2.28 \times 2.28 \times 0.033 \mathrm{~mm}$ diaphragm with three rectangular rigid islands for stress concentration in four $0.490 \times 0.041 \mathrm{~mm}$ grooves separating either rigid islands or rigid islands and the frame (see Fig. 4d). The microstructure is formed by wet anisotropic etching.

Similar to the PDA circuit, the piezotresistors are formed by combination of low-doped $\mathrm{P}^{-}$areas and high-doped $\mathrm{P}^{+}$areas. Transistors and piezoresistors are electrically isolated by isolation diffusion. P-substrate is connected to ground. Difference in electrical potentials of collectors of transistors $T_{1}$ and $T_{2}$ is used as output signal $\Delta U$ of the sensor. The NDA-NFL circuit is biased by constant voltage of $5.0 \mathrm{~V}$. Target values of piezoresistors and operating points of BJTs are listed in Table 1.

\section{Table 1}

Parameters of PDA-NFL circuit

\begin{tabular}{cccc}
\hline & \multirow{2}{*}{ Parameters } & \multicolumn{2}{c}{ Electrical circuits } \\
& V-NPN & L-PNP \\
\hline \multirow{3}{*}{ Bipolar } & Base current $\mathrm{I}_{\mathrm{b}}, \mu \mathrm{A}$ & 4.6 & 44.0 \\
junction & Gain $\beta$ & 145 & 5 \\
transistor & Emitter-base voltage drop $\mathrm{U}_{\mathrm{em}-\mathrm{b}}, \mathrm{V}$ & 0.80 & 0.77 \\
& Collector-base voltage drop $\mathrm{U}_{\mathrm{c}-\mathrm{b}}, \mathrm{V}$ & 0.80 & 0.86 \\
& Collector potential $\mathrm{U}_{\mathrm{c}}, \mathrm{V}$ & 2.79 & 2.81 \\
& Parasitic substrate current $\mathrm{I}_{\mathrm{sub}}, \mu \mathrm{A}$ & - & 30.0 \\
Resistors & $\mathrm{R}_{\mathrm{b} 11, \mathrm{~b} 21}, \mathrm{KOhm}$ & 4.47 & 3.00 \\
& $\mathrm{R}_{\mathrm{b} 12, \mathrm{~b} 22, \mathrm{KOhm}}$ & 2.98 & 2.00 \\
& $\mathrm{R}_{\mathrm{c} 1, \mathrm{c} 2}, \mathrm{KOhm}$ & 3.33 & 10.00 \\
& $\mathrm{R}_{\mathrm{em} 1, \mathrm{em} 2}, \mathrm{KOhm}$ & 1.79 & 1.50 \\
\hline
\end{tabular}

Parasitic current from emitter to substrate should be taken into account in the circuit utilizing L-PNP transistors. This current exists even when $\mathrm{p}-\mathrm{n}$ junction between epitaxial layer and substrate is closed. This current has been taken into account at the design phase and modeled in Sentaurus TCAD software. This parasitic substrate current can be as high as $30 \mu \mathrm{A}$ when base current $\mathrm{I}_{\mathrm{b} 1,2}=44 \mu \mathrm{A}$ (about $2 / 3$ of base current) - see Table 1 .

Performance of sensor chip with PDA-NFL circuit has been compared both with sensor chip utilizing PDA circuit and with the standard pressure sensor product IPD60 based on Wheatstone bridge circuit formed by four piezoresistors. IPD60 has the same mechanical structure of diaphragm as PDA-NFL chip and fabricated on n-type DSP (100) silicon wafers using a similar process. Table 2 provides average values of performance parameters for all four pressure sensor chips powered by constant voltage of $5.0 \mathrm{~V}$ and for the differential pressure range of $60 \mathrm{kPa}$ applied from the cavity side. 
Performance over temperature was measured in two ranges: $-30 \ldots+20^{\circ} \mathrm{C}$ and $+20 \ldots+80^{\circ} \mathrm{C}$. Pressure overload testing was done on both sides of the sensor chips by applying pressure of $250 \mathrm{kPa}$. Static pressure testing was performed at $25 \mathrm{MPa}$. Stability of output signal was monitored during the first nine hours after powering on the sensors. Output signal noise was measured as root mean square deviation of output signal from its average value during 20 minutes. Sensor chips of all types were assembled in metal packages with silicon stress-relief pedestals protecting sensor chip from packaging stress. Bonding of sensor chip to the stress-relief pedestal was performed using low-temperature glass.

Sensor chip PDA-NFL also has been characterized in a narrower temperature range of $-30 \ldots+60^{\circ} \mathrm{C}$. It was done because BJTs with high gain $\beta$ may enter saturation regime at elevated temperature [19]. It was found that performance of PDA-NFL sensor chip is significantly better in the narrower temperature range (see Table 3 ).

Table 2

Performance of pressure sensors chips: PDA-NFL, PDA and IPD60

\begin{tabular}{|c|c|c|c|c|c|}
\hline \multirow{2}{*}{\multicolumn{2}{|c|}{ Parameter }} & \multicolumn{4}{|c|}{ Sensor Chip } \\
\hline & & $\begin{array}{l}\text { PDA-NFL } \\
\text { (V-NPN) }\end{array}$ & $\begin{array}{l}\text { PDA-NFL } \\
\text { (L-PNP) }\end{array}$ & IPD60 & PDA \\
\hline \multicolumn{2}{|c|}{ Sensitivity $\mathrm{S}, \mathrm{mV} / \mathrm{V} / \mathrm{kPa}$} & 1.877 & 1.216 & 0.515 & 0.667 \\
\hline \multicolumn{2}{|c|}{ Zero pressure output signal (Offset), $\mathrm{mV} / \mathrm{V}$} & 6.8 & 9.2 & 3.0 & 6.6 \\
\hline \multicolumn{2}{|c|}{ Noise voltage, $\mu \mathrm{V}$} & 15 & 60 & 4 & 300 \\
\hline \multicolumn{2}{|c|}{ Nonlinearity $2 \mathrm{~K}_{\mathrm{NL}}, \% \mathrm{FS}$} & 0.059 & 0.051 & 0.055 & 0.105 \\
\hline \multicolumn{2}{|c|}{$\operatorname{TCZ}\left(-30 \ldots+20^{\circ} \mathrm{C}\right), \% \mathrm{FS} /{ }^{\circ} \mathrm{C}$} & 0.007 & 0.051 & 0.003 & \\
\hline \multicolumn{2}{|c|}{$\mathrm{TCZ}\left(+20 \ldots+80^{\circ} \mathrm{C}\right), \% \mathrm{FS} /{ }^{\circ} \mathrm{C}$} & 0.019 & 0.056 & 0.002 & $\approx 0.250$ \\
\hline \multicolumn{2}{|c|}{$\mathrm{TCS}\left(-30 \ldots+20^{\circ} \mathrm{C}\right), \% \mathrm{FS} /{ }^{\circ} \mathrm{C}$} & 0.020 & 0.005 & 0.043 & \\
\hline \multicolumn{2}{|c|}{$\operatorname{TCS}\left(+20 \ldots+80^{\circ} \mathrm{C}\right), \% \mathrm{FS} /{ }^{\circ} \mathrm{C}$} & 0.091 & 0.011 & 0.034 & $\approx 0.32$ \\
\hline \multirow{2}{*}{$\begin{array}{l}\text { Zero thermal } \\
\text { hysteresis }\end{array}$} & $\left(-30 \ldots+20^{\circ} \mathrm{C}\right), \% \mathrm{FS}$ & 0.065 & 0.252 & 0.033 & \multirow{2}{*}{$\approx 1.030$} \\
\hline & $\left(+20 \ldots+80^{\circ} \mathrm{C}\right), \% \mathrm{FS}$ & 0.077 & 0.538 & 0.025 & \\
\hline \multirow{2}{*}{$\begin{array}{l}\text { Span thermal } \\
\text { hysteresis }\end{array}$} & $\left(-30 \ldots+20^{\circ} \mathrm{C}\right), \% \mathrm{FS}$ & 0.042 & 0.063 & 0.018 & \multirow{2}{*}{$\approx 0.330$} \\
\hline & $\left(+20 \ldots+80^{\circ} \mathrm{C}\right), \% \mathrm{FS}$ & 0.020 & 0.040 & 0.012 & \\
\hline \multirow{2}{*}{$\begin{array}{l}\text { Output } \\
\text { change }\end{array}$} & after pressure overload, \%FS & 0.006 & 0.006 & 0.004 & - \\
\hline & under static pressure, \%FS & 0.069 & 0.072 & 0.072 & - \\
\hline \multirow{2}{*}{$\begin{array}{l}\text { Long-term } \\
\text { instability }\end{array}$} & of offset, \%FS & 0.007 & 0.008 & 0.002 & - \\
\hline & of pressure sensitivity, \%FS & 0.004 & 0.006 & 0.003 & - \\
\hline \multicolumn{2}{|c|}{ Number of samples in statistics } & 20 & 28 & 1200 & 12 \\
\hline
\end{tabular}

Table 3

Some parameters of sensor chip with PDA-NFL (V-NPN) circuit tested up to $80^{\circ} \mathrm{C}$ and $60^{\circ} \mathrm{C}$

\begin{tabular}{lcc}
\hline \multicolumn{1}{c}{ Parameter } & \multicolumn{2}{c}{ Maximum Test Temperature } \\
\cline { 2 - 3 } & $\mathrm{T}_{+}=+60{ }^{\circ} \mathrm{C}$ & $\mathrm{T}_{+}=+80{ }^{\circ} \mathrm{C}$ \\
\hline $\mathrm{TCZ}\left(+20 \ldots+60 /+80{ }^{\circ} \mathrm{C}\right), \% \mathrm{FS} /{ }^{\circ} \mathrm{C}$ & 0.010 & 0.019 \\
$\mathrm{TCS}\left(+20 \ldots+60 /+80^{\circ} \mathrm{C}\right), \% \mathrm{FS} /{ }^{\circ} \mathrm{C}$ & 0.045 & 0.091 \\
Zero thermal hysteresis $\left(+20 \ldots+60 /+80{ }^{\circ} \mathrm{C}\right), \% \mathrm{FS}$ & 0.045 & 0.077 \\
Span thermal hysteresis $\left(+20 \ldots+60 /+80^{\circ} \mathrm{C}\right), \% \mathrm{FS}$ & 0.017 & 0.020 \\
\hline
\end{tabular}


Temperature dependence of zero output signal shows that sensitivity / span starts to change above $+60^{\circ} \mathrm{C}$ and BJT enters saturation regime at $\mathrm{T}>90^{\circ} \mathrm{C}$ (see Fig. 6).

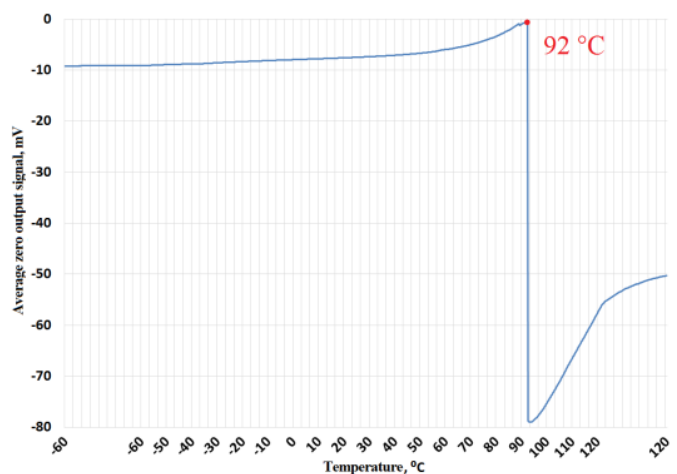

a)

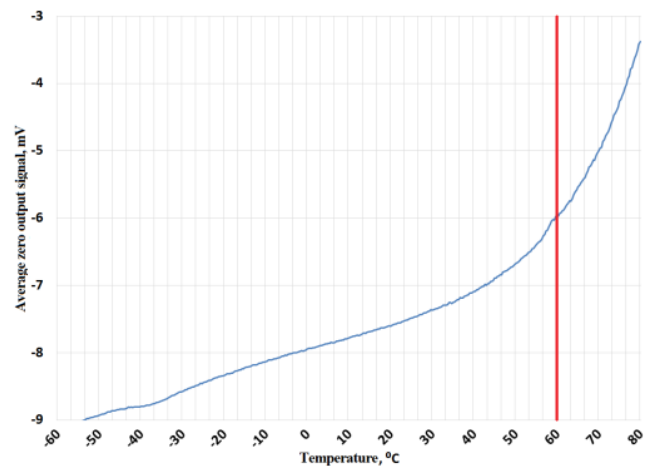

b)

Fig. 6. Dependence of average zero output signal on temperature for PDA-NFL (V-NPN) in temperature ranges of: a) $\left.-60 \ldots+120^{\circ} \mathrm{C}, \mathrm{b}\right)-60 \ldots+80^{\circ} \mathrm{C}$.

\section{Discussion}

The following conclusions can be made based on experimental data on performance of pressure sensor with PDA-NFL circuit utilizing V-NPN BJTs:

- The main advantage of the novel pressure sensor chip is significant sensitivity increase while keeping the same die size and the same geometry of the diaphragm. Sensitivity increased by factor of 3.6 compared to IPD60 sensor chip and by factor of 2.8 compared to sensor chip with PDA circuit.

- Sensor chip with PDA-NFL circuit has output signal noise about 20 times lower compared to prototypes with PDA circuit. However, output signal noise of PDA-NFL circuit is higher than output signal noise of IPD60 sensor. In case of ASIC-assisted signal conditioning, somewhat higher output signal noise of sensor chip may be not critical.

- Temperature dependence of zero output signal and sensitivity in sensor chip with PDA-NFL circuit is improved by about one order of magnitude compared to prototypes with PDA circuit. Sensor chip with PDA-NFL (V-NPN) circuit still has 2-3 times higher temperature errors than that of IPD60 sensor chip. Reduction of temperature errors and opening up the operating temperature range requires some additional work and better understanding of factors affecting temperature instability of PDA-NFL circuit. It is likely that some reduction of temperature errors can be achieved by optimizing both values of piezoresistors and operating points of transistors. Currently, sensor chip with PDA-NFL (V-NPN) circuit can be used in operating temperature range up to $60^{\circ} \mathrm{C}$. At higher temperatures, performance of the circuit may be affected by one of V-NPN BJTs entering saturation regime and by growing leakage current.

Sensor chip with PDA-NFL (L-PNP) circuit has 2.4 times higher sensitivity compared to IPD60 sensor chip, 5 times smaller noise compared to prototypes with TDA circuit. This sensor chip has pressure non-linearity error, pressure overload range and static 
pressure error comparable with both PDA-NFL (V-NPN) and IPD60 sensor chips. Low TCS value, which potentially can be decreased to be zero, is the only advantage of L-PNP circuit compared to V-NPN circuit. All other parameters of PDA-NFL sensor with V-NPN circuit are better than corresponding parameters of PDA-NFL sensor with L-PNP circuit.

Fig. 7 shows clear advantage of sensor chip with PDA-NFL V-NPN circuit over IPD52 sensor chip utilizing traditional piezoresistive bridge circuit: die size is reduced (about 2.4X area reduction) and pressure overload capability is increased.
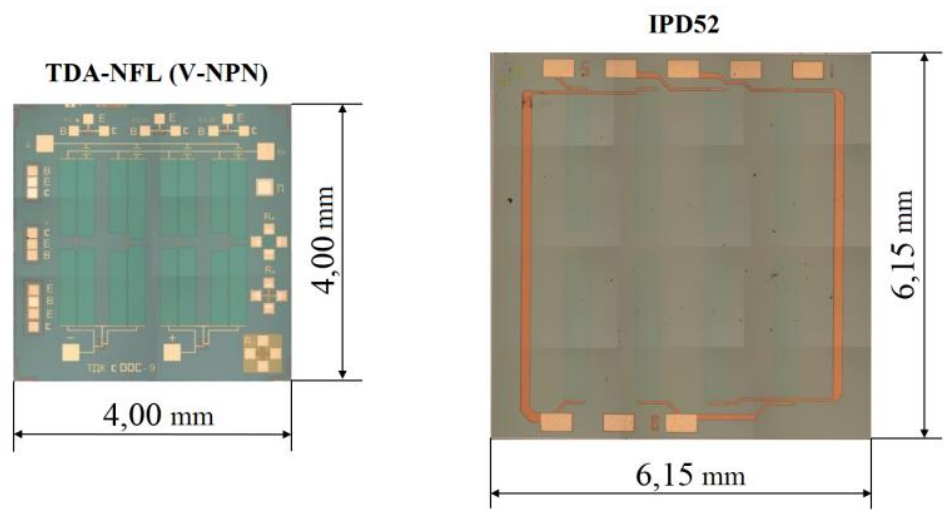

Fig. 7. Pressure sensor chips: PDA-NFL with V-NPN circuit (left) and IPD52 (right).

Sensor chip with PDA-NFL (V-NPN) circuit has been compared with IPD52 pressure sensor chip. IPD52 chip utilizes Wheatstone bridge circuit. IPD52 and IPD60 chips share the same manufacturing processes. IPD52 die size is $6.15 \mathrm{~mm} \times 6.15 \mathrm{~mm}$. It has 4,20 $\mathrm{mm} \times 4,20 \mathrm{~mm} \times 0.035 \mathrm{~mm}$ diaphragm with three rigid islands. Chip with PDA-NFL (V-NPN) circuit has the same pressure sensitivity as IPD52 (about $1.9 \mathrm{mV} / \mathrm{V} / \mathrm{kPa}$ ) although it is 2.4 times smaller area and has 5 times higher burst pressure of $1.6 \mathrm{MPa}$.

\section{Conclusion}

Testing of the three types of developed pressure sensors utilizing BJT confirmed possibility of significant increase of pressure sensitivity while maintaining the same die size and diaphragm size. Two novel circuits for piezoresistive MEMS sensors have been introduced and tested. Both circuits use differential amplifier based on two bipolar junction transistors. One of the circuits (PDA-NFL) utilizes negative feedback loop while the other circuit (PDA) does not have it. Two types of bipolar junction transistors have been tested: vertical n-p-n transistors (V-NPN) and horizontal p-n-p transistors (L-PNP). Modeling of PDA-NFL circuit showed that placement of BJTs in the areas with the maximum stress provides only limited increase in pressure sensitivity (1\% to $5 \%$ ).

Sensor chip with PDA circuit. The first pressure sensor with PDA circuit utilizing V-NPN BJTs allows for 2.2X sensitivity increase compared to pressure sensor having similar mechanical structure but utilizing Wheatstone bridge circuit formed by piezoresistors. However, temperature errors and output noise of this sensor chip are high.

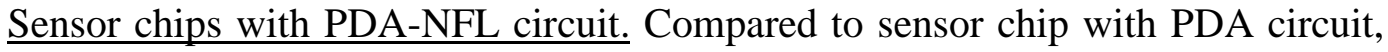


electrical circuit used in PDA-NFL chip allows for combining larger number of piezoresistors. PDA-NFL circuit design - specifically selection of target values of resistors - allows for compensation of temperature dependence of electrical potential of transistor base. With the same mechanical design of both die and diaphragm, pressure sensitivity of sensor chip with PDA-NFL circuit is much higher than pressure sensitivity of IPD60 sensor chip. The sensitivity increase is 3.6X for circuit utilizing V-NPN transistors and 2.4X for circuit utilizing L-PNP transistors. Sensor chip with V-NPN BJTs and balanced values of piezoresistors allows for smaller values of measurement errors. Compared to PDA circuit, PDA-NFL circuit has 10+ times smaller temperature errors. In fact, the temperature errors are close to that of "traditional" chip IPD60. Output signal noise of PDA-NFL circuit is only about $15 \mu \mathrm{V}$, which is about 20 times smaller compared to PDA circuit. Besides that, sensor chips utilizing PDA-NFL circuit have 2-3 better stability. Pressure nonlinearity, pressure overload and static pressure errors are close to parameters of similar pressure sensor chips utilizing Wheatstone bridge circuit. The demonstrated approach can be used for chip size reduction and increase of pressure overload capability while maintaining the same pressure sensitivity.

Described in this paper sensor chip utilizing novel PDA-NFL circuit based on two BJTs is a perspective direction for development of advanced piezoresistive sensors.

\section{Acknowledgment}

The studies were financial supported by Dukhov Automatics Research Institute.

\section{Abbreviations}

PDA-NFL - piezosensitive differential amplifier with negative feedback loop.

\section{References}

[1] Y. Charentenay, MEMS Pressure Sensor Market and Technologies 2018, I-Micronews. Market \& Technology report (2018) 28-31.

[2] Hafez, N., Haas, S., Loebel, K., 2018. Characterisation of MOS Transistors as an Electromechanical Transducer for Stress. Physica Status Solidi A. 1700680.

[3] X. Zhao, D. Wen, G. Li, Fabrication and Characteristics of an nc-Si/c-Si Heterojunction MOSFETs Pressure Sensor, Sensors 12 (2012) 6369-6379.

[4] P. Gieschke, Y. Nurcahyo, M. Herrmann, M. Kuhl, P. Ruther, O. Paul. CMOS Integrated Stress Mapping Chips with $32 \mathrm{~N}$-type or P-type Piezoresistive Field Effect Transistors, 2009 IEEE 22nd International Conference on MicroElectroMechanical Systems (2009) 769-772.

[5] P. Singh, J. Miao, L. Shao, Microcantilever sensors with embedded piezoresistive transistor read-out: Design and characterization, Sensors and Actuators A: Physical 171 (2011) 178-185. 
[6] W. Rindner, G. Doering, Structural and Operational Characteristics of Piezo-Transistor and Allied Devices, Solid-State Electronics 8 (1965) 227-240.

[7] Y. Kanda, Y. Kanazawa, T. Terada, M.Maki, Improved Si Piezo-Transistors for Mechano-Electrical Transducers, IEEE Transactions on Electron Devices ED-25 (1978) 813-817.

[8] R. Edwards, Some Effect of Localized Stress on Silicon Planar Transistor, IEEE Transactions on Electron Devices 11 (1964) 286-294.

[9] A.L. Polyakova, Deformation of Semiconductors and Semiconductor Devices, Energy, Moscow, 1979 (in Russian).

[10] V.V. Beklemishev, V.I. Vaganov, A.V. Sumin, Integral Pressure Transducers Based on Planar and Longitudinal Strain Transistors, in: A.G. Filippov (Eds.), Electronic measuring equipment, Atomizdat, Moscow, 1978, pp. 124-130 (in Russian).

[11] V.I. Vaganov, Intergrated Piezoresistive Sensors, Energoatomizdat, Moscow, 1983 (in Russian).

[12]F. Fruett, G.C.M. Meijer, The Piezojunction Effect in Silicon Integrated Circuits and Sensors, Springer, Delft, 2002.

[13] L. Li, N. Belov, M. Klitzke, J-S. Park, High Performance Piezoresistive Low Pressure Sensors, IEEE Sensors Conference (2016) 1406-1408.

[14] Tran, A.V., Zhang, X., Zhu, B., 2018. Mechanical Structural Design of a Piezoresistive Pressure Sensor for Low-Pressure Measurement: A Computational Analysis by Increases in the Sensor Sensitivity. Sensors. 18, 2023.

[15] Xu, T., Lu, D., Zhao, L., Jiang, Z., Wang., H., Guo, X., Li, Z., Zhou, X., Zhao, Y., 2017. Application and Optimization of Stiffness Abruption Structures for Pressure Sensors with High Sensitivity and Anti-Overload Ability. Sensors. 17, 1965.

[16] T. Guan, F. Yang, W. Wang, X. Huang, B. Jiang, D. Zhang, The Design and Analysis of Piezoresistive Shuriken-Structured Diaphragm Micro-Pressure Sensors, Journal of Microelectromechanical Systems 26 (2017) 206-214.

[17] M.V. Basov, D.M. Prigodskiy, Investigation of a Sensitive Element for the Pressure Sensor Based on a Bipolar Piezotransistor, Nano- and Microsystem Technology 19 (2017) 685-693.

[18] M.V. Basov, D.M. Prigodskiy, D.A. Holodkov, Modeling of Sensitive Element for Pressure Sensor Based on Bipolar Piezotransistor, Sensors and Systems 6 (2017) 17-24.

[19] B.M. Wilamowski, J.D. Irwin, The industrial Electronics Handbook. Fundamentals of industrial electronics, Taylor and Francis Group, Boca Raton, 2011.

\section{Biographies}

Basov Mikhail graduated in 2012 from National Nuclear Research University "Moscow Physics Engineering Institute" (department of Nano- and Microelectronics). Starting from 2010 he works as R\&D engineer on advanced MEMS pressure sensing elements. His Ph.D. thesis is titled "High-Sensitivity Pressure Sensing Chip Utilizing Bipolar-Junction Transistors". 